\title{
Cultura tributaria y el cumplimiento del pago del rus de los microempresarios de la Urb. Mirasol II, Jaén - 2020
}

\author{
Br. Leyla Maribel Coronel Facundo \\ lcoronelfa@ucvvirtual.edu.pe \\ https://orcid.org/0000-0003-4084-0306
}

\author{
Br. Lady Lozada Chamaya \\ $\underline{\text { llozadach@ucvvirtual.edu.pe }}$ \\ https://orcid.org/0000-0001-5070-1946
}

Mg. Roberto Carlos Oblitas Otero

https://orcid.org/0000-0002-6541-7005

roblitas@ucvvirtual.edu.pe

Universidad César Vallejo

Moyobamba - Perú

\section{RESUMEN}

Se decidió proponer como propósito principal: Determinar cómo incide la Cultura tributaria en el cumplimiento del pago del RUS de los microempresarios de la Urb. Mirasol II, Jaén - 2020. Asimismo, se manejó una investigación de diseño no experimental, de corte transversal, con enfoque cuantitativo, de tipo aplicada y nivel correlacional causal, la población estuvo compuesta por 350 microempresarios de la Urb. Mirasol II, Jaén, aplicándose el cuestionario a 85 microempresarios de la misma urbanización. Los resultados evidenciaron que el valor del Rho de Spearman obtenido es de 0.543 y el valor de la Sig., es menor al 0,05, llegando a concluir que el nivel de incidencia de la cultura tributaria es positiva moderada con el cumplimiento de pago, esto revela que, mientras los microempresarios tengan mayor cultura tributaria entonces se podría decir que se incrementa el cumplimiento fiscal.

Palabras clave: valores tributarios; conocimiento tributario; actitudes tributarias; sujeto pasivo. 


\title{
Tax culture and compliance with the payment of the rus of the microentrepreneurs of Urb. Mirasol II, Jaen - 2020
}

\begin{abstract}
It was decided to propose as main purpose: To determine how the tax culture affects the compliance with the payment of the RUS of the microentrepreneurs of Urb. Mirasol II, Jaen - 2020. Likewise, a non-experimental research design, cross-sectional, with quantitative approach, applied and causal correlational level, the population was composed of 350 microentrepreneurs of Mirasol II, Jaén, and the questionnaire was applied to 85 microentrepreneurs of the same urbanization. The results showed that the value of Spearman's Rho obtained is 0.543 and the value of the Sig. is less than 0.05 , concluding that the level of incidence of tax culture is moderately positive with payment compliance, this reveals that, while microentrepreneurs have a greater tax culture, it could be said that tax compliance increases.
\end{abstract}

Key words: tax values; tax knowledge; tax attitudes; taxpayer

Artículo recibido: 05 octubre. 2021 Aceptado para publicación: 02 noviembre 2021 Correspondencia: 1coronelfa@ucvvirtual.edu.pe

Conflictos de Interés: Ninguna que declarar 


\section{INTRODUCCIÓN}

Los ciudadanos de las naciones más avanzadas en la tierra, poseen una cultura tributaria muy alta, permitiendo a estas naciones contar con los recursos económicos suficientes que les permite enfrentar y cubrir las necesidades de cada persona (Boong \& Chang, 2021). Todo esto sucede porque sus autoridades no cuentan con privilegios personales por ser funcionarios públicos, y porque son transparentes al momento de rendir cuentas por la ejecución de los gastos gubernamentales que llevan a cabo (Qi \& Che, 2021). De acuerdo al CEPAL (2019), el cobro de impuestos a los microempresarios del Caribe en el 2019 cayó a un -49\%; en Colombia la percepción de los impuestos disminuyó en un 54\% (Secretaria Distrital de Hacienda, 2020); y en México no fue la excepción, donde el Gobierno informó que en el 2020 la recaudación de los impuestos a los microempresarios ha caído en un -62\% (Lux Consultores en comercio y Desarrollo , 2019); demostrándose que los sujetos pasivos no cuentan con una cultura tributaria eficiente, trayendo como consecuencia un déficit fiscal para todos estos países, impidiéndoles enfrentar la crisis que ha golpeado enormemente la economía mundial (Mawani \& Umashanker, 2021).

En el aspecto nacional, en palabras de López (2020), menciona que, el aumento de la informalidad ha ocasionado que la recaudación de los impuestos de los microempresarios que se localizan en el NRUS disminuya, ya que, estos tipos de sujetos pasivos tributan sobre ingresos y no sobre resultados, por lo que estas empresas no cuentan con incentivos para exigir comprobantes de pago y de esta forma puedan deducir el gasto, costo y crédito fiscal, pese que los contribuyentes de este régimen se incrementaron a 1,2 millones en el 2018, ha caído la recaudación de impuestos en un -47\% en ese mismo año (Arias, 2018). De acuerdo a la SUNAT (2021), la recaudación de impuestos de los sujetos pasivos del Régimen Único Simplificado, cayó en -7,3\% en marzo, recuperándose en abril 2020 con un aumento del $3,4 \%$, pero de mayo a diciembre del mismo año cayó $-9 \%$ en la recaudación de los microempresarios de este régimen fiscal.

Con respecto a los microempresarios de la ciudad de Jaén, se evidencian como problemas principales es la no emisión de boletas de pago por día, donde estos tienen la obligación tributaria de emitir un comprobante de pago, donde deben considerar todos los ingresos del día, esto afecta considerablemente a los intereses de la SUNAT, ya que, solo cumplen con pagar S/ 20 mensuales, pese que de acuerdo a sus niveles de ingreso deben cancelar $\mathrm{S} / 50$, incluso hay unos que ya deben cambiarse a un régimen tributario más alto; estos 
factores evidencian que los microempresarios buscan de cualquier forma no pagar impuestos elevados, dejando de lado los intereses de un país, por priorizar los suyos.

\section{A. Trabajos previos}

Atuguba (2021). Los resultados obtenidos evidenciaron que sí, el Gobierno logra vincular la cultura tributaria a la gobernanza administrativa, de forma práctica y concreta con la tributación, podrán aumentar el cobro de impuestos que en los últimos tiempos ha mermado sus ingresos. Hantono (2021). En su investigación determinaron que el conocimiento tributario, la conciencia tributaria y la moral tributaria alivian el cumplimiento tributario. Demostrando que el conocimiento tributario, tiene menos impacto en el cumplimiento tributario y no se reconoce la moral tributaria. Hassan et al. (2021). Las derivaciones encontradas revelaron que, la evidencia tiene un impacto mayor en la gestión fiscal que la percepción sobre el gasto público y la moral físcal. Además, la percepción de equidad media significativa en las fortalezas entre la moral, la simplicidad, el gasto público y el comportamiento de cumplimiento. Sharma (2021). Las implicaciones políticas sugieren que la solicitud de un mayor cumplimiento tributario debe basarse en la creación de un ecosistema en el que se aspira a pagar impuestos a largo plazo. Korostelkina et al. (2020). El proceso de interacción entre el estado y los contribuyentes (el nivel de desarrollo de las relaciones tributarias) y determinar el grado de protección de los intereses nacionales y sostenible desarrollo. Gerba (2020). La evasión fiscal se derivaba de falta de conocimiento sobre tributación, percibiendo la evasión fiscal como cultura, tendencia de la fiscalización tributaria y el grado de detección, percepción tributaria la evasión como delito menor y cuestiones relacionadas con la equidad y equidad tributaria. Asgharpour et al. (2019). Después de analizar los resultados, se evidenció que cada uno de los componentes que integran la cultura fiscal de los contribuyentes fue superior a satisfactoria, esto quiere decir, que el aumento de la recaudación se debe al alto nivel de cultura tributaria de los sujetos pasivos de este país. Nurwanah (2018). El comportamiento de los contribuyentes posee un efecto positivo y significativo en la intención de gravar cumplimiento, esto quiere decir, que se conseguiría una mayor recaudación de impuestos si el Gobierno Indonesio les brindara la importancia adecuada a estos aspectos.

Con respecto a los antecedentes del ámbito nacional, se consideró el estudio de Quispe et al. (2021). Al final del trabajo los autores evidenciaron que, existe una correlación fuerte 
entre ambas variables que se investigaron en el artículo, revelándose que mientras la entidad recaudatoria realice programas de concientización tributaria, permitirá aumentar la cultura fiscal de los sujetos obligados impactando positivamente en el cumplimiento de pago. Ramírez y Nolazco (2020). Que es probable que una mejora en la imagen de las autoridades y en la transparencia de las administraciones públicas pueda resultar importante para mejorar el cumplimiento voluntario de los contribuyentes. Todos los hechos mencionados deben ser confirmados mediante estudios específicos. Condori et al. (2020). Determinaron que, al ejecutar el evento didáctico aumentaron los conocimientos, apreciaciones y cualidades providenciales permitiendo el pago voluntario de sus tributos al Estado.

Quispe et al. (2020). Los factores que afectan en el cumplimiento del pago tributario, es la baja cultura tributaria, el nivel de educación y el nivel de ingreso familiar mensual. Cárdenas (2020). El acatamiento de los compromisos tributarios requiere que los contribuyentes verifiquen determinadas obligaciones formales según el sistema tributario seleccionado, y se sancionará su cumplimiento parcial o tardío. Si bien se han establecido los supuestos de determinación y control, su incumplimiento se ha convertido en una forma diferente de práctica evasiva y ha dado lugar a impuestos nacionales. Impacto negativo. Hinojosa (2019). El nivel de conocimientos de estas personas es bajo, siendo el factor determinante que sigan en la informalidad y no cumplan con el pago de sus tributos. Luján y Cano (2019). La variable 1 incide elocuentemente en el procedimiento de los delitos tributarios, donde a mayor cultura tributaria menor es el número de delitos tributarios. Ruíz (2017). El nivel de relación entre ambas variables es significativa fuerte, donde el Sig., es menor que 0,05 y el Rho de Spearman es de 0,451, demostrándose que a mayor cultura tributaria aumenta la recaudación de impuestos.

\section{B. Teorías relacionadas al tema}

El enfoque considerado es la teoría de la renta - producto, donde Alva (2017), pretende explicar las supuestas afectaciones en el pago de los impuestos, ya que, la renta es un producto, porque contienen un periodo determinado y proviene de fuentes duraderas en el tiempo, esto quiere decir, que mientras mayores ingresos tengan las organizaciones, mayor será el pago de los impuestos. Estos impuestos son calculados mensualmente, a través de la presentación de registros y libros contables electrónicos que la entidad recaudadora ha aprobado para que todas las empresas cumplan con la presentación de su 
declaración jurada mensual, de la misma forma, existen sujetos pasivos que están obligados a presentar declaraciones juradas de forma anual (Nurlela et al., 2021).

En el cumplimiento tributario, se tomó en cuenta la teoría de las decisiones, donde Gutiérrez (2017), explica que cada segundo las personas toman alguna decisión para llevar a cabo alguna acción en el aspecto familiar, personal o profesional, siendo en algunas oportunidades negativas, que no solo afectan el estado emocional, sino que tienen la obligación de buscar alguna solución si en el caso desean conseguir lo que se han proyectado. En esta teoría se hace mención que las personas tomamos decisiones por lo que observamos, escuchamos, por la experiencia o por comentarios de algún familiar, amigo o vecino, es por ello que, el Estado debe ser transparente en todas las ejecuciones de presupuesto público, debiendo impactar positivamente en el bienestar de la sociedad, ya que, de esto depende que la gran mayoría de personas puedan cumplir o incumplir el pago de sus impuestos (McKee, 2020).

La cultura tributaria, es el conocimiento que se tiene de los métodos tributarios que le permita identificar de forma clara y precisa qué impuestos, cómo y cuándo los debe cancelar (Superintendencia de Administración Tributaria, 2019). Asimismo, es el grupo de sapiencias, apreciaciones, cualidades y habilidades de conducta de una colectividad respecto a la tributación (SAT, 2017). Se entiende, como el grupo de supuestos primordiales de conducta de una metrópoli que asume su compromiso con analogía al pago de tributos (García, 2017).

Las dimensiones consideradas en la cultura tributaria son el conocimiento tributario, valores tributarios y actitudes tributarias.

Con respecto a la dimensión 1, de la cultura tributaria, es el conocimiento tributario, donde Mollocondo (2017), indica que el Gobierno debe priorizar en brindar información a los ciudadanos sobre todos los ingresos tributarios que este ha logrado recaudar, ya que, a través de esta mecánica se demuestra que las autoridades están siendo totalmente transparentes, esto ayudaría a que se aumente el compromiso en el pago real de los impuestos que los sujetos pasivos deben cumplir fehacientemente.

La dimensión 2, de la variable cultura tributaria, son los valores tributarios, donde Mollocondo (2017), manifiesta que mientras siga existiendo actos de corrupción en tres niveles de gobierno, incluso en la entidad que supuestamente fiscaliza y audita a las entidades públicas, no se va a recuperar la confianza de los ciudadanos, es por ello que, 
se debe promover a todas las jurisdicciones de todos los niveles de gobierno a impulsar los valores tributarios, tratando por igual si eres una empresa nacional o extranjera, o si eres micro o gran empresa. Es de vital importancia dar el ejemplo, para que todos los ciudadanos que se encuentran obligados a tributar lo hagan voluntariamente, pero tomando en cuenta todos sus ingresos obtenidos por día, haciendo una sumatoria mensual para posteriormente presentarlo en la declaración jurada (Bin et al., 2020).

La dimensión 3, de la variable cultura tributaria, son las actitudes tributarias, hace mención que las personas rechazan tajantemente los actos de corrupción, pero existe cierta cantidad de personas que respaldan la corrupción, como muestra tenemos a dos candidatos a la presidencia con equipos técnicos que han sido sancionados por actos de enriquecimiento ilícito logrados por los actos de corrupción, son estas formas que merman la voluntad en el pago de los impuestos de la gran mayoría de contribuyentes, prefiriendo seguir siendo informal (Mollocondo, 2017).

El cumplimiento tributario, es una correlación legal “ex lege", toda persona está forzada hacia el Estado, al pago de una suma de dinero, en cuanto se verifique el presupuesto de hecho determinado por la ley (Código Tributario, 1989). Asimismo, es la presentación de la declaración jurada de gravámenes en término, conteniendo información completa y correcta a fin de decretar el deber tributario (Centro Interamericano Administraciones Tributarias, 2010).

Posterior a las citas de los autores, se procedió a consultar a diferentes profesionales sobre su apreciación de un concepto de cumplimiento tributario, donde el magister y contador público colegiado Víctor Hugo Puican Rodríguez, docente de diferentes universidades nacionales y con amplia experiencia profesional, nos mencionó que el cumplimiento tributario, es el acto económico que realizan los sujetos pasivos de todas sus obligaciones tributarias adquiridas por las actividades empresariales que estos realizan a través de la venta de sus productos o la prestación de servicios, debiendo tomarse en cuenta el régimen tributario que estas se encuentran.

Las dimensiones del cumplimiento tributario son las obligaciones sustanciales y las obligaciones formales.

La dimensión 1, de la variable 2 cumplimiento tributario, son las obligaciones sustanciales, donde la SUNAT (2019). Revela que las cuotas mensuales que pagan los contribuyentes bajo el nuevo sistema único simplificado se establecen en función de sus 
compras y / o ingresos, en lugar del pago de varios impuestos. El sistema está dirigido a personas físicas que venden bienes o servicios a consumidores finales. Quienes desarrollan el comercio también pueden beneficiarse de la nueva RUS. El sistema tributario permite la emisión de tickets de venta y tickets de caja como comprobante de pago. Para este tipo de sujetos pasivos está prohibido emitir facturas y otros comprobantes que otorguen créditos fiscales, y existen restricciones sobre los ingresos máximos y / o compras mensuales y / o anuales y el desarrollo de algunas actividades prohibidas. (SUNAT, 2019).

La dimensión 2, de la variable cumplimiento tributario, son las obligaciones formales, donde la SUNAT (2019), indica que, quienes inician actividades durante el año fiscal solo son elegibles cuando están inscritos en el Registro Único de Contribuyentes. Sin embargo, si están involucrados contribuyentes del sistema general, sistema especial o RMT, solo se pueden cambiar durante la declaración y pago en enero de cada año. Además, deben realizar los siguientes pasos para que la aceptación de NRUS sea válida. Debes realizarla antes del 31 de diciembre del año anterior al que deseas postular. El retiro de facturas o cualquier otro comprobante de pago físico permite mantener los impuestos de crédito, costos y / o el formato de los gastos, como liquidación de compra, documentos de autorización, etc. (SUNAT, 2019).

Debiendo darle de baja de las filiales anexas autorizadas y sobre todo declarar y costear la cuota correspondiente al periodo de enero del año en que se va a acoger, esta declaración y pago se deberá presentar dentro de la fecha de vencimiento de acuerdo al último dígito del RUC (SUNAT, 2019).

\section{ESTRATEGIAS METODOLÓGICAS O MATERIALES Y MÉTODOS}

Hemos decidido emplear una investigación de tipo aplicada, donde Hernández (2018), menciona que es una exploración activa que se encuentra totalmente atada a la anterior, porque obedece a los hallazgos y aportes teóricos. Asimismo, se consideró manejar un estudio correlacional causal; ya que, posee como principio medir el nivel de relación entre las variables y sus dimensiones (Vásquez, 2020). Se consideró como objetivo general, determinar cómo incide la Cultura tributaria en el cumplimiento del pago del RUS de los microempresarios de la Urb. Mirasol II, Jaén - 2020.

Para encontrar la relación de ambas variables, primero se utilizó la prueba de normalidad, donde por poseer una muestra superior a 50 microempresarios se utilizó el Kolmogorov- 
Smirnov, obteniendo una significancia menor a 0.05, siendo la razón por el cual se utilizó el coeficiente de correlación de Rho de Spearman.

Se utilizó el diseño de investigación no experimental, debido a que no se manipuló ninguna de las variables estudiadas, de tal manera el estudio será analizado tal como se muestra en el contexto empresarial en un periodo determinado.

Se presentó el tema de investigación al docente del taller de tesis, después de su aprobación, se dio inicio al desarrollo del primer capítulo, después que fue aprobado, se continuo con el desarrollo del marco teórico, el cual, está compuesto por los enfoques científicos, conceptos y dimensiones de cada variable de estudio, posterior a ello, se elaboró la metodología, para que, después de su aprobación elaborar el cuestionario, para que sea validado por tres expertos, siendo dos contadores y un profesional en educación para la parte metodológica, todos los expertos cuentan con grado de magister y con amplia experiencia profesional y académica. Se siguió con la aplicación de la encuesta, permitiendo agregar dichos datos al Excel, para su traslado al SPSS vr. 26, el cual, nos permitió obtener los resultados para su posterior análisis e interpretación, llegando a las consumaciones y recomendaciones.

\section{RESULTADOS Y DISCUSIÓN}

\subsection{RESULTADOS}

\section{Tabla 1.}

Incidencia de la cultura tributaria con el cumplimiento del pago del RUS de los microempresarios de la Urb. Mirasol II, Jaén-2020

\begin{tabular}{|c|c|c|c|c|}
\hline & & & $\begin{array}{c}\text { Cultura } \\
\text { tributaria }\end{array}$ & $\begin{array}{c}\text { Cumplimiento } \\
\text { de pago }\end{array}$ \\
\hline \multirow{8}{*}{$\begin{array}{l}\text { Rho de } \\
\text { Spearman }\end{array}$} & \multirow{4}{*}{ Cultura tributaria } & Coeficiente de & 1,000 &, $543^{* *}$ \\
\hline & & correlación & & \\
\hline & & Sig. (bilateral) & &, 000 \\
\hline & & $\mathrm{N}$ & 85 & 85 \\
\hline & \multirow{4}{*}{ Cumplimiento de pago } & Coeficiente de &, $543^{* *}$ & 1,000 \\
\hline & & correlación & & \\
\hline & & Sig. (bilateral) & ,000 & . \\
\hline & & $\mathrm{N}$ & 85 & 100 \\
\hline
\end{tabular}

**. La correlación es significativa al nivel 0,01 (bilateral).

De acuerdo a las derivaciones reflejadas en la Tabla 1, se evidencia que el valor del Rho de Spearman obtenido es de 0.543 y el valor de la Sig., es menor al 0,05, demostrándose que el nivel de incidencia de la cultura tributaria es positiva moderada con el cumplimiento tributario. 


\section{Tabla 2.}

Nivel de incidencia de la cultura tributaria en el conocimiento tributario con el cumplimiento del pago del RUS de los microempresarios de la Urb. Mirasol II, Jaén2020

\begin{tabular}{lllcc}
\hline & & & $\begin{array}{c}\text { Conocimiento } \\
\text { tributario }\end{array}$ & $\begin{array}{c}\text { Cumplimiento } \\
\text { de pago }\end{array}$ \\
\hline & $\begin{array}{l}\text { Conocimiento } \\
\text { tributario }\end{array}$ & $\begin{array}{l}\text { Coeficiente } \\
\text { correlación }\end{array}$ & 1,000 &, $525^{* *}$ \\
Rho de & & Sig. (bilateral) & & \\
Spearman & & $\mathrm{N}$ & 85 &, 001 \\
& & Coeficiente de &, $525^{* *}$ & 85 \\
& Cumplimiento de pago & correlación & & 1,000 \\
& & Sig. (bilateral) &, 001 & \\
& & $\mathrm{~N}$ & 85 & 100 \\
\hline
\end{tabular}

\section{**. La correlación es significativa al nivel 0,01 (bilateral).}

De acuerdo a las derivaciones reflejadas en la Tabla 2, se evidencia que el valor del Rho de Spearman obtenido es de 0.525 y el valor de la Sig., es menor al 0,05, demostrándose que el nivel de incidencia del conocimiento es positiva moderada con el cumplimiento tributario, esto revela que, mientras los sujetos pasivos aumenten su conocimiento tributario, aumenta el cumplimiento fiscal. Siendo aceptable la hipótesis de investigación, rechazando la hipótesis nula.

\section{Tabla 3.}

Nivel de incidencia de la cultura tributaria en los valores tributarios con el cumplimiento del pago del RUS de los microempresarios de la Urb. Mirasol II, Jaén - 2020

\begin{tabular}{|c|c|c|c|c|}
\hline & & & $\begin{array}{c}\text { Valores } \\
\text { Tributarios }\end{array}$ & $\begin{array}{c}\text { Cumplimiento } \\
\text { de pago }\end{array}$ \\
\hline \multirow{6}{*}{$\begin{array}{l}\text { Rho de } \\
\text { Spearman }\end{array}$} & \multirow{3}{*}{ Valores Tributarios } & $\begin{array}{l}\text { Coeficiente de } \\
\text { correlación }\end{array}$ & 1,000 & $406^{* *}$ \\
\hline & & Sig. (bilateral) & &, 000 \\
\hline & & $\mathrm{N}$ & 85 & 85 \\
\hline & \multirow{3}{*}{ Cumplimiento de pago } & $\begin{array}{l}\text { Coeficiente de } \\
\text { correlación }\end{array}$ &, $406^{* *}$ & 1,000 \\
\hline & & Sig. (bilateral) &, 000 & \\
\hline & & $\mathrm{N}$ & 85 & 100 \\
\hline
\end{tabular}

\section{**. La correlación es significativa al nivel 0,01 (bilateral).}

De acuerdo a las derivaciones reflejadas en la Tabla 3, se evidencia que el valor del Rho de Spearman obtenido es de 0.406 y el valor de la Sig., es menor al 0,05, demostrándose que el nivel de incidencia de los Valores Tributarios es positiva moderada con el cumplimiento tributario, esto revela que, esto revela que, mientras los sujetos pasivos 
tomen en cuenta los valores tributarios aumenta el cumplimiento fiscal. Siendo aceptable la hipótesis de investigación, rechazando la hipótesis nula.

\section{Tabla 4.}

Nivel de incidencia de la cultura tributaria en las actitudes tributarias con el cumplimiento del pago del RUS de los microempresarios de la Urb. Mirasol II, Jaén2020

\begin{tabular}{|c|c|c|c|c|}
\hline & & & $\begin{array}{c}\text { Actitudes } \\
\text { Tributarias }\end{array}$ & $\begin{array}{c}\text { Cumplimiento } \\
\text { de pago }\end{array}$ \\
\hline \multirow{5}{*}{$\begin{array}{l}\text { Rho de } \\
\text { Spearman }\end{array}$} & \multirow[t]{2}{*}{ Actitudes Tributarias } & $\begin{array}{l}\text { Coeficiente de } \\
\text { correlación } \\
\text { Sig. (bilateral) }\end{array}$ & 1,000 &, $625^{* *}$ \\
\hline & & $\mathrm{N}$ & 85 & 85 \\
\hline & \multirow{3}{*}{ Cumplimiento de pago } & $\begin{array}{l}\text { Coeficiente de } \\
\text { correlación }\end{array}$ &, $625^{* *}$ & 1,000 \\
\hline & & Sig. (bilateral) & ,000 & 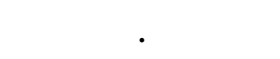 \\
\hline & & $\mathrm{N}$ & 85 & 100 \\
\hline
\end{tabular}

**. La correlación es significativa al nivel 0,01 (bilateral).

De acuerdo a las derivaciones reflejadas en la Tabla 4, se evidencia que el valor del Rho de Spearman obtenido es de 0,625 y el valor de la Sig., es menor al 0,05, demostrándose que el nivel de incidencia de las actitudes tributarias es positiva moderada con el cumplimiento tributario, esto revela que, mientras los sujetos pasivos tomen actitudes tributarias positivas y de acorde a ley entonces aumenta el cumplimiento fiscal. Siendo aceptable la hipótesis de investigación, rechazando la hipótesis nula.

\section{Tabla 5}

Nivel de cultura tributaria de los microempresarios de la Urb. Mirasol II, Jaén-2020

\begin{tabular}{lcccccc}
\hline \multirow{2}{*}{ Secciones } & \multicolumn{2}{c}{ Bajo } & \multicolumn{2}{c}{ Regular } & \multicolumn{2}{c}{ Alto } \\
& f & \% & f & \% & f & \% \\
\hline Cultura tributaria & 27 & 31.8 & 52 & 61.2 & 6 & 7.1 \\
Conocimiento tributario & 55 & 64.7 & 30 & 35.3 & 0 & 0 \\
Valores tributarios & 67 & 78.8 & 18 & 21.2 & 0 & 0 \\
Actitudes tributarias & 49 & 57.6 & 27 & 31.8 & 9 & 10.6 \\
\hline
\end{tabular}

Se observa que la variable cultura tributaria alcanzó un $61.2 \%$ en el nivel regular y el 31.8 en el nivel bajo, en cambio, sus dimensiones presentaron diferentes niveles, la que presenta mayores dificultades son los valores tributarios porque alcanzó un $78.8 \%$ en el nivel bajo, seguidamente por el conocimiento tributario porque alcanzó un $64.7 \%$ en el nivel bajo y por último tenemos a las actitudes tributarias que alcanzó un 57.6\%. 


\section{Tabla 6}

Nivel del cumplimiento del pago de los microempresarios de la Urb. Mirasol II, Jaén2020

\begin{tabular}{lcccccc}
\hline \multirow{2}{*}{ Secciones } & \multicolumn{2}{c}{ Bajo } & \multicolumn{2}{c}{ Regular } & \multicolumn{2}{c}{ Alto } \\
& f & \% & f & \% & f & \% \\
\hline Cumplimiento de pago & 26 & 30.6 & 59 & 69.4 & 0 & 0 \\
Obligaciones sustanciales & 25 & 29.4 & 18 & 21.2 & 42 & 49.4 \\
Obligaciones formales & 32 & 37.6 & 22 & 25.9 & 31 & 36.5 \\
\hline
\end{tabular}

Según la Tabla 6, se observa que la variable cumplimiento de pago alcanzó un $69.4 \%$ en el nivel regular y el 30.6 en el nivel bajo, en cambio, sus dimensiones presentaron diferentes niveles, la que presenta mayores dificultades son las obligaciones formales porque alcanzó un $37.6 \%$ en el nivel bajo, en cambio, las obligaciones sustanciales alcanzaron un 49.4 en el nivel alto, teniendo más de la mitad en niveles bajo y regular.

\subsection{DISCUSIÓN}

Para fortalecer el trabajo de investigación las autoras se ampararon en el enfoque de la renta - producto, donde Alva (2017), esta teoría nos permitió conocer los tiempos que poseen los microempresarios para presentar sus declaraciones juradas, conocer la periodicidad de cálculo de sus impuestos y el monto que deben cancelar, tomando como referencia todas las actividades económicas que han generado ingresos de recursos financieros a la empresa. Asimismo, se tomó en cuenta la teoría de las decisiones, de Gutiérrez (2017), este enfoque ayudo a determinar que los seres humanos tomamos decisiones en algunas ocasiones por lo que observamos y escuchamos, esto quiere decir que, mientras las autoridades sean transparentes en la difusión de la información de ejecución de presupuesto público, las personas cumplirán voluntariamente con el pago de sus impuestos.

De acuerdo al objetivo general, se evidencia que el valor del Rho de Spearman obtenido es de 0.543 y el valor de la Sig., es menor al 0,05, demostrándose que el nivel de incidencia de la cultura tributaria es positiva moderada con el cumplimiento tributario. Siendo aceptable la hipótesis de investigación, rechazando la hipótesis nula. Estos resultados son amparados por el estudio de Atuguba (2021). Logró determinar que los indicadores que afectan negativamente a la cultura tributaria es la deficiente gobernanza administrativa, porque al no realizarla de forma práctica y concreta afecta en el cumplimiento fiscal. Asimismo, lo respalda la investigación de Hinojosa (2019). El factor 
que determina una baja en la cultura tributaria, es el nivel de conocimientos de estas personas sobre el uso de los recursos económicos, siendo el factor determinante que sigan en la informalidad y no cumplan con el pago de sus tributos.

De acuerdo al primer objetivo específico, se evidencia que el valor del Rho de Spearman obtenido es de 0.525 y el valor de la Sig., es menor al 0,05, demostrándose que el nivel de incidencia del conocimiento es positiva moderada con el cumplimiento tributario, esto revela que, mientras los sujetos pasivos aumenten su conocimiento tributario, aumenta el cumplimiento fiscal. Siendo aceptable la hipótesis de investigación, rechazando la hipótesis nula. Estos datos son respaldados por el trabajo de Cárdenas (2020). El acatamiento de los compromisos tributarios requiere que los contribuyentes verifiquen determinadas obligaciones formales según el régimen tributario seleccionado, y se sancionará su cumplimiento parcial o tardío. Si bien se han establecido los supuestos de determinación y control, su incumplimiento se ha convertido en una forma diferente de práctica evasiva y ha dado lugar a impuestos nacionales. Impacto negativo; de la misma forma lo hace el estudio de Hassan et al., (2021). En esta investigación se determinó que la evidencia de los gastos ejecutados tiene un impacto mayor en la gestión fiscal. Además, la percepción de equidad media significativa en las fortalezas entre la moral, la simplicidad, el gasto público y el comportamiento de cumplimiento.

De acuerdo al segundo objetivo específico, se evidencia que el valor del Rho de Spearman obtenido es de 0.406 y el valor de la Sig., es menor al 0,05, demostrándose que el nivel de incidencia de los Valores Tributarios es positiva moderada con el cumplimiento tributario, esto revela que, esto revela que, mientras los sujetos pasivos tomen en cuenta los valores tributarios aumenta el cumplimiento fiscal. Siendo aceptable la hipótesis de investigación, rechazando la hipótesis nula. Estas derivaciones son salvaguardadas por la investigación de Hantono (2021). Los indicadores que generan una cultura tributaria alta en los microempresarios es el conocimiento tributario, la conciencia tributaria y la moral tributaria ya que, esto permite aliviar el cumplimiento tributario. De la misma forma lo hace el estudio de Quispe et al., (2020). En este caso se evidenció que los factores que afectan en el cumplimiento del pago tributario, es la baja cultura tributaria, el nivel de educación y el nivel de ingreso familiar mensual.

De acuerdo al tercer objetivo específico, se evidencia que el valor del Rho de Spearman obtenido es de 0,625 y el valor de la Sig., es menor al 0,05, demostrándose que el nivel 
de incidencia de las actitudes tributarias es positiva moderada con el cumplimiento tributario, esto revela que, mientras los sujetos pasivos tomen actitudes tributarias positivas y de acorde a ley entonces aumenta el cumplimiento fiscal. Siendo aceptable la hipótesis de investigación, rechazando la hipótesis nula. Se amparan en el trabajo de Korostelkina et al., (2020). Los indicadores que permiten mantener una cultura fiscal positiva, es el proceso de interacción entre el estado y los contribuyentes y determinar el grado de protección de los intereses nacionales y sostenible desarrollo. Y en la investigación de Ruíz (2017). El nivel de relación entre ambas variables es significativa fuerte, donde el Sig., es menor que 0,05 y el Rho de Spearman es de 0,451, demostrándose que a mayor cultura tributaria aumenta la recaudación de impuestos.

De acuerdo al cuarto objetivo específico, se observa que la variable cultura tributaria alcanzó un $61.2 \%$ en el nivel regular y el $31.8 \%$ en el nivel bajo, en cambio, sus dimensiones presentaron diferentes niveles, es así que, la dimensión con mayores dificultades son los valores tributarios porque alcanzó un $78.8 \%$ en el nivel bajo, seguidamente por el conocimiento tributario porque alcanzó un $64.7 \%$ en el nivel bajo y por último tenemos a las actitudes tributarias que alcanzó un $57.6 \%$. Estos datos son respaldados por el trabajo de Quispe et al., (2020), porque evidenciaron que los factores que afectan en el cumplimiento del pago tributario, es la baja cultura tributaria, el nivel de educación y el nivel de ingreso familiar mensual.

De acuerdo al quinto objetivo específico, se observa que la variable cumplimiento de pago alcanzó un $69.4 \%$ en el nivel regular y el 30.6\% en el nivel bajo, en cambio, sus dimensiones presentaron diferentes niveles, es así que, la dimensión con mayores dificultades son las obligaciones formales porque alcanzó un 37.6\% en el nivel bajo, en cambio, las obligaciones sustanciales alcanzaron un $49.4 \%$ en el nivel alto, teniendo más de la mitad en niveles bajo y regular. Estos datos son respaldados por la investigación de Sharma, A. y Singh, J. (2021), porque evidenciaron que las implicaciones políticas sugieren que la solicitud de un mayor cumplimiento tributario debe basarse en la creación de un ecosistema en el que se aspira a pagar impuestos a largo plazo.

Asimismo, lo hace el trabajo de Nurwanah, A. (2018), porque demostró que el comportamiento de los contribuyentes posee un efecto positivo y significativo en la intención de gravar cumplimiento, esto quiere decir, que se conseguiría una mayor recaudación de impuestos si el Gobierno Indonesio les brindara la importancia adecuada 
a estos aspectos. Del mismo modo lo hace el estudio de Ramírez, A. y Nolasco, J. (2020), porque revelaron que es probable que una mejora en la imagen de las autoridades y en la transparencia de las administraciones públicas pueda resultar importante para mejorar el cumplimiento voluntario de los contribuyentes.

\section{CONCLUSIÓN O CONSIDERACIONES FINALES}

- Se concluye que el valor del Rho de Spearman obtenido es de 0.543 y el valor de la Sig., es menor al 0,05, demostrándose que el nivel de incidencia de la cultura tributaria es positiva moderada con el cumplimiento tributario.

- Se concluye que el valor del Rho de Spearman obtenido es de 0.525 y el valor de la Sig., es menor al 0,05, demostrándose que el nivel de incidencia del conocimiento es positiva moderada con el cumplimiento tributario, esto revela que, mientras los sujetos pasivos aumenten su conocimiento tributario, aumenta el cumplimiento fiscal.

- Se concluye que el valor del Rho de Spearman obtenido es de 0.406 y el valor de la Sig., es menor al 0,05, demostrándose que el nivel de incidencia de los Valores Tributarios es positiva moderada con el cumplimiento tributario, esto revela que, esto revela que, mientras los sujetos pasivos tomen en cuenta los valores tributarios aumenta el cumplimiento fiscal.

- Se concluye que el valor del Rho de Spearman obtenido es de 0,625 y el valor de la Sig., es menor al 0,05, demostrándose que el nivel de incidencia de las actitudes tributarias es positiva moderada con el cumplimiento tributario, esto revela que, mientras los sujetos pasivos tomen actitudes tributarias positivas y de acorde a ley entonces aumenta el cumplimiento fiscal.

- Se identificó que la variable cultura tributaria alcanzó un $61.2 \%$ en el nivel regular y el 31.8 en el nivel bajo, en cambio, sus dimensiones presentaron diferentes niveles, la que presenta mayores dificultades son los valores tributarios porque alcanzó un $78.8 \%$ en el nivel bajo, seguidamente por el conocimiento tributario porque alcanzó un $64.7 \%$ en el nivel bajo y por último tenemos a las actitudes tributarias que alcanzó un $57.6 \%$.

- Se identificó que la variable cumplimiento de pago alcanzó un $69.4 \%$ en el nivel regular y el 30.6 en el nivel bajo, en cambio, sus dimensiones presentaron diferentes niveles, la que presenta mayores dificultades son las obligaciones formales porque 
alcanzó un 37.6\% en el nivel bajo, en cambio, las obligaciones sustanciales alcanzaron un 49.4 en el nivel alto, teniendo más de la mitad en niveles bajo y regular.

\section{LISTA DE REFERENCIAS}

Alva, M. (2017). El impuesto a la renta y las teorías que determinan su afectación. http://blog.pucp.edu.pe/blog/blogdemarioalva/2012/03/01/el-impuesto-a-larenta-y-las-teorias-que-determinan-su-afectacion/

Arias, L. (2018). El Perú hacia la OCDE La agenda pendiente para la política tributaria 2018-2021.

http://cooperaccion.org.pe/wp-content/uploads/2018/09/17071-El-Peru-hacia-laOCDE-CORR-web.pdf

Asgharpour, L., Pourkiani, M., \& Addolmajid, S. (2019). Investigating tax culture of the tax payers of the iranian tax administration. Revista Inclusiones, 6, 17. http://revistainclusiones.org/pdf38/11\%20VOL\%206\%20NUM\%203\%202019D OSESPASIAJULIOSEPTIEMBREEASIIAEUROPAASIA19INCL.pdf

Atuguba, R. A. (2021). Tax Culture: Perspectives from an African State. Research House $a b c, 8(1), 25-58.10 .18034 / a j t p . v 8 i 1.510$. https://abc.us.org/ojs/index.php/ajtp/article/view/510/1002

Bin, S., Al-Hamedi, A.-M., Marimuthu, M., \& Al-Harethi, A. (2020). Study on system fairness dimensions and tax compliance in the Middle East context. Business Perspectives, 18(1), 181-191. http://dx.doi.org/10.21511/ppm.18(1).2020.16.

Boong, H., \& Chang, Y. (2021). Determinants of Tax Compliance Intention: Focus on the Theory of Planned Behavior. International Journal of Public Administration, 44(1), 62-73.

https://doi.org/10.1080/01900692.2020.1728313.

Cárdenas, G. (2020). La educación tributaria en el Perú: una alternativa para mejorar el cumplimiento de las obligaciones tributarias. In Crescendo, 11(2), 241-258. http://dx.doi.org/10.21895/incres .

Centro Interamericano Administraciones Tributarias. (2010). Serie temática tributaria. https://www.ciat.org/Biblioteca/SerieTematica/Espanol/2010_n6_dic_cumplimi ento_trib.pdf 
CEPAL. (2019). Panorama fiscal de ameríca latina y el Caribe: Políticas tributarias para la movilización de recursos en el marco de la agenda 2030 para el desarrollo

sostenible. https://www.cepal.org/sites/default/files/publication/files/44516/S1900075_es.p df

Código Tributario. (1989). Texto Único Ordenado del Código Tributario. http://www.oas.org/juridico/pdfs/mesicic3_per_tributario.pdf

Condori, S., Mamani, O., \& Bernedo, D. H. (2020). Preferencias eficacia del programa ¡Qué importante es tributar! en el desarrollo de cultura tributaria en adolescentes. Revista de Investigación, $10(1), \quad 1-11$. https://www.redalyc.org/jatsRepo/4676/467663403005/467663403005.pdf

Condori, S., Mamani, O., \& Bernedo, D. H. (2020). Preferencias eficacia del programa ¡Qué importante es tributar! en el desarrollo de cultura tributaria en adolescentes. Apuntes $\quad$ universitarios, $\quad 10(1), \quad 1-11$. https://www.redalyc.org/jatsRepo/4676/467663403005/467663403005.pdf

Consejo Nacional de Ciencia, Tecnología e Innovación Tecnológica. (2018). Reglamento de calificación, clasificación y registro de los Investigadores del sistema nacional de ciencia, tecnología e innovación tecnológica - Reglamento RENACYT. Lima, Perú:

CONCYTEC. https://portal.concytec.gob.pe/images/renacyt/reglamento_renacyt_version_final. pdf

García, J. (2017). Elevemos la cultura tributaria en el Perú. https://www.uch.edu.pe/uch-noticias/p/elevemos-la-cultura-tributaria-en-el-peru

Gerba, B. (2020). Factors Affecting Perception of Taxpayers towards the Seriousness of Tax Evasion in Bale Robe Town Administration, Oromia, Ethiopia. International Journal of Finance and Accounting, 9(2), 21-30.

10.5923/j.ijfa.20200902.01.

Gutierrez, G. (2017). Teoría de la toma de decisiones. Definición, etapas y tipos. https://www.gestiopolis.com/teoria-de-la-toma-de-decisiones-definicion-etapasy-tipos/ 
Hantono. (2021). The impact tax knowledge, tax awareness, tax morale toward tax compliance boarding house tax. International Journal of Research, 9(1), 49-65. https://doi.org/10.29121/granthaalayah.v9.i1.2021.2966.

Hassan, I., Naeem, A., \& Gulzar, S. (2021). Voluntary tax compliance behavior of individual taxpayers in Pakistan. Financial Innovation, 7(21), 23. https://doi.org/10.1186/s40854-021-00234-4.

Hernández. (2018). El proceso de la investigación cientídica (4ta ed.). LIMUSA Noriega Editores.

Hinojosa, C. A. (2019). Cultura tributaria para el incremento de los ingresos a los comerciantes del mercado modelo, Chachapoyas, 2018. Revista Científica UNTRM, 2(1), 22-26.

http://dx.doi.org/10.25127/rcsh.20192.463.

Korostelkina, I., Dedkova, E., Varaksa, N., \& Korostelkin, M. (2020). Models of tax relations: improving the tax culture and discipline of taxpayers in the interests of sustainable development. Web of Conferences, 159, 13. https://doi.org/10.1051/e3sconf/202015906014.

López, L. (2020). Es ilegal que el ejecutivo modifique la cuantía de los impuestos. https://lacamara.pe/leonardo-lopez-es-ilegal-que-el-ejecutivo-modifique-lacuantia-de-los-impuestos/

Luján, R. M., \& Cano, E. A. (2019). La Cultura Tributaria y el Comportamiento de los Delitos Tributarios en el Perú: Periodo 2012-2016. Revista Ciencia y Tecnología, 15(2), 101-109.

https://revistas.unitru.edu.pe/index.php/PGM/article/view/2378

Lux Consultores en comercio y Desarrollo . (2019). Impuesto predial, análisis y alternativas para mejorar la capacidad recaudatoria en los municipios de México.

http://www.cefp.gob.mx/transp/CEFP-CEFP-70-41-C-Estudio0009-010617.pdf

Mawani, A., \& Umashanker, V. (2021). Collusive vs. coercively corrupt tax auditors and their impact on tax compliance. $30,1-14$. https://doi.org/10.1016/j.jbef.2021.100470.

McKee, M. (2020). High Tax Compliance Results in Smaller Government. Springer Link, 40, 67-73. 
https://doi.org/10.1007/978-3-030-55081-3_4.

Mollocondo, L. (2017). Cultura tributaria en los stands de la galeria Los Inkas y su incidencia en la contribución de la oblligación tributaria - periodo 2016. [Tesis de pregrado, Universidad Privada San Carlos]. http://repositorio.upsc.edu.pe/bitstream/handle/UPSC/4397/Liliana_MOLLOCO NDO_FLORES.pdf?sequence $=1 \&$ isAllowed $=\mathrm{y}$

Nurlela, I., Kuriniawan, A., \& Umiyati, I. (2021). The effect of awareness, morality, tax culture, and distributive justice on the taxpayer compliance. Accounting Research Journal of $\quad$ Sutaatmadja, $\quad 5(1), \quad 112-129$. https://doi.org/10.35310/accruals.v5i01.699.

Nurwanah, A. (2018). Determinants of tax compliance: theory of planned behavior and stakeholder theory perspective. Business Perspectives, 16(4), 395-407. http://dx.doi.org/10.21511/ppm.16(4).2018.33.

Qi, Y., \& Che, A. (2021). Factors affecting electronic invoice adoption and tax compliance process efficiency. Discover Journals, 15(1), 150-168. https://doi.org/10.1108/TG-04-2020-0070.

Quispe, A. A., Gutierrez, E. W., \& Maldonado, L. A. (2021). Study on the tax culture and compliance with the obligations of the Arenales food market in the city of Ica. Journal of Global Management Sciences, 4(1), 7-16. https://doi.org/10.32829/gms.v4i1.216.

Quispe, J. C., Guevara, M., Roque, C. E., Marca, H. R., \& Marca, V. R. (2020). Factores que influyen en el cumplimiento del pago del Impuesto al valor del patrimonio predial en la ciudad de Puno - Perú. Ciencia Latina Revista Multidisciplinar, 4(2), 268-285.

https://doi.org/10.37811/cl_rcm.v4i2.75.

Ramírez, A., \& Nolazco, J. L. (2020). Assessment of fiscal effort and voluntary tax compliance in Peru. Revista de Finanzas y Política Económica, 12(1), 55-87. 10.14718/revfinanzpolitecon.v12.n1.2020.3121.

Ruíz, J. (2017). La cultura tributaria y la gestión municipal. Quipukamayoc, 25(48), 4960. http://dx.doi.org/10.15381/quipu.v25i48.13992.

Ruíz, J. (2017). Tax culture and municipal management. QUIPUKAMAYOC, 25(48), 4960. http://dx.doi.org/10.15381/quipu.v25i48.13992. 
Sánchez, A., Revilla, D., Alayza, M., Sime, L., Trelles, L., \& Tafur, R. (2020). Métodos de investigación para la elaboración de las tesis de maestría en educación (1ra ed.). Pontificia Universidad Católica del Perú. https://files.pucp.education/facultad/educacion/wpcontent/uploads/2020/08/03180404/LIBRO-LOS-M\%C3\%89TODOS-DEINVESTIGACI\%C3\%93N-MAESTR\%C3\%8DA-2020.pdf

SAT. (2017).

Cultura tributaria.

http://www.educacionfiscal.org/files/documentos/Cultura\%20Tributaria\%20$\% 2050 \% 20$ preguntas\%20y\%20respuestas.pdf

Secretaria Distrital de Hacienda. (2020). Niveles de recaudación del impuesto predial. https://economia.uniandes.edu.co/sites/default/files/webproyectos/comisionstt/C ESTT-Informe-web.pdf

Sharma, A., \& Singh, J. (2021). Analysing the dynamics of tax attitude: a study of potential taxpayers. International Journal of Indian Culture and Business Management, 17.

https://doi.org/10.1504/IJICBM.2021.114991.

Sharma, A., \& Singh, J. (2021). Analysing the dynamics of tax attitude: a study of potential taxpayers. International Journal of Indian Culture and Business Management, 1-17.

https://doi.org/10.1504/IJICBM.2021.114991.

SUNAT. (2019). Nuevo RUS.

http://orientacion.sunat.gob.pe/index.php/empresas-menu/nuevo-regimen-unicosimplificado-nuevo-rus

SUNAT. (2021). Ingresos recaudados por la SUNAT. https://www.sunat.gob.pe/estadisticasestudios/index.html

Superintendencia de Administración Tributaria. (2019). Cultura Tributaria. https://portal.sat.gob.gt/portal/cultura-tributaria/

Vásquez, W. A. (2020). Metodología de la investigación. https://www.usmp.edu.pe/estudiosgenerales/pdf/2020-

I/MANUALES/II\%20CICLO/METODOLOGIA\%20DE\%20INVESTIGACION .pdf 